\title{
Biomarker microRNAs for prostate cancer metastasis: screened with a network vulnerability analysis model
}

Yuxin Lin ${ }^{1}$, Feifei Chen ${ }^{1}$, Li Shen ${ }^{1,2}$, Xiaoyu Tang ${ }^{1,3}$, Cui Du ${ }^{1}$ Zhandong Sun ${ }^{1,4}$, Huijie Ding ${ }^{1,5}$, Jiajia Chen ${ }^{6}$ and Bairong Shen ${ }^{1,7,8^{*}}$ (1)

\begin{abstract}
Background: Prostate cancer ( $\mathrm{PCa}$ ) is a fatal malignant tumor among males in the world and the metastasis is a leading cause for PCa death. Biomarkers are therefore urgently needed to detect PCa metastatic signature at the early time. MicroRNAs are small non-coding RNAs with the potential to be biomarkers for disease prediction. In addition, computer-aided biomarker discovery is now becoming an attractive paradigm for precision diagnosis and prognosis of complex diseases.
\end{abstract}

Methods: In this study, we identified key microRNAs as biomarkers for predicting PCa metastasis based on network vulnerability analysis. We first extracted microRNAs and mRNAs that were differentially expressed between primary PCa and metastatic PCa (MPCa) samples. Then we constructed the MPCa-specific microRNA-mRNA network and screened microRNA biomarkers by a novel bioinformatics model. The model emphasized the characterization of systems stability changes and the network vulnerability with three measurements, i.e. the structurally single-line regulation, the functional importance of microRNA targets and the percentage of transcription factor genes in microRNA unique targets.

Results: With this model, we identified five microRNAs as putative biomarkers for PCa metastasis. Among them, miR-101-3p and miR-145-5p have been previously reported as biomarkers for PCa metastasis and the remaining three, i.e. miR-204-5p, miR-198 and miR-152, were screened as novel biomarkers for PCa metastasis. The results were further confirmed by the assessment of their predictive power and biological function analysis.

Conclusions: Five microRNAs were identified as candidate biomarkers for predicting PCa metastasis based on our network vulnerability analysis model. The prediction performance, literature exploration and functional enrichment analysis convinced our findings. This novel bioinformatics model could be applied to biomarker discovery for other complex diseases.

Keywords: Bioinformatics model, Network vulnerability analysis, MicroRNA biomarkers, Prostate cancer metastasis

\footnotetext{
*Correspondence: bairong.shen@suda.edu.cn

${ }^{1}$ Center for Systems Biology, Soochow University, Suzhou 215006, China

Full list of author information is available at the end of the article
} 


\section{Background}

Prostate cancer ( $\mathrm{PCa}$ ) is one of the common malignant tumors worldwide. In western countries, it has become the second major cause of cancer death among men [1]. The incidence and mortality of this disease were also increasing in Asia during the last decade [2]. At present, the degree of PCa is stratified as low- or high-risk based on the Gleason score, prostate-specific antigen level and other clinical indices. However, such classification seems to be insufficient to monitor PCa progression, especially at the time of metastasis initiation [3]. The early detection of PCa metastatic signature is crucial for evaluating $\mathrm{PCa}$ outcome, therefore screening key molecules as biomarkers for predicting PCa metastases is of clinical significance.

MicroRNAs are a group of endogenous, small noncoding RNAs with approximately 22-24 nucleotides in length [4]. They regulate gene expression through baseparing with target messenger RNAs (mRNAs) at the posttranscriptional level and thereby play important roles in a number of important cellular processes [5]. Extensive efforts have been made to identify reliable microRNAs as biomarkers because microRNAs are remarkably stable and specific to be detected in tissues, blood as well as other bodily fluids [6-8]. The expression level of circulating microRNAs also exhibits characteristic alteration in individuals with different pathological settings [9].

Currently, substantial investigations are devoted to discovering microRNA biomarkers for PCa metastasis evaluation, most of which are experimental. Firstly the differentially expressed (DE) or dysregulated microRNAs from large-scale microRNA expression data were extracted as outliers and then the candidates were further validated by low-throughput experiments, such as realtime PCR, etc. $[3,10,11]$. Although experimental methods are powerful enough to detect the abnormal change of microRNA expression between different condition groups, e.g., primary $\mathrm{PCa}$ (PPCa) and metastatic $\mathrm{PCa}$ (MPCa) [12], it is not easy to identify the driver or key molecules at the systems level. As we known, the mechanism of PCa metastasis is complex, where the dysregulation of microRNAs tends to be highly heterogeneous due to the genetic and environmental factors [13, 14].

Nowadays, computational approaches based on systems biology and network science are well performed on detecting microRNA or gene signatures for the diagnosis and treatment of complex diseases such as cancers $[15,16]$, diabetes [17] and neurodevelopmental disorders [18]. In particular, Zhang et al. introduced a correlation and clustering based framework to identify microRNAmRNA network modules for differentiating PPCa and MPCa subtypes [19]. We here aim to develop a novel bioinformatics method to screen single microRNA biomarkers for predicting PCa metastasis, which is propitious to PCa prognosis and therapy.

In our previous studies, we proposed a network-based bioinformatics model called Pipeline of Outlier MicroRNA Analysis (POMA) to detect microRNA biomarkers for cancer diagnosis and prognosis [20-22]. The model integrates microRNA/mRNA expression data with the structural information of microRNA-mRNA regulatory network. Two measurements, i.e., number of single-line regulation (NSR, the number of genes that uniquely regulated by a single microRNA) and transcription factor (TF) gene percentage (TFP, the percentage of TF genes targeted by a single microRNA), were defined to quantify the regulatory power of independent microRNAs. These features characterize network systems vulnerability because the abnormal change of unique regulatory relationships cannot be compensated by other interactions. Statistical evidences demonstrated that microRNAs with significantly high NSR and TFP values are more likely to be biomarkers [20, 21]. In this study, we improved and updated the model as MicroRNA Biomarker Discovery (MicroRNA-BD) by analyzing the network vulnerability and considering the functional importance of genes that are uniquely regulated by given microRNAs. We then applied the model to screen key microRNAs as biomarkers for predicting PCa metastasis. The schematic pipeline of the MicroRNA-BD model is shown in Fig. 1.

\section{Methods \\ Data collection}

The microRNA and mRNA expression dataset for biomarker prediction (GSE21036 [23] and GSE3325 [24]) were downloaded from gene expression omnibus (GEO) [25]. Among them, GSE21036 was generated by Agilent-019118 Human microRNA Microarray 2.0 G4470B, and contained microRNA expression data from 142 prostate tissue samples. Here 113 of the samples including 99 PPCa and 14 MPCa were extracted for further analysis. The mRNA expression dataset GSE3325 was performed on Affymetrix Human Genome U133 Plus 2.0 Array, 5 and 4 individual samples for PPCa and $\mathrm{MPCa}$ were selected, respectively. Moreover, another independent dataset GSE26964 [26] with 6 PPCa and 7 MPCa samples from Capitalbio mammal microRNA V3.0 platform was selected for result validation. The details are summarized in Table 1.

Besides, the previously reported PCa microRNA biomarkers were manually collected and integrated from: (1) the review for PCa microRNAs by Vanacore et al. [27], (2) NCBI PubMed using the retrieval terms as "(prostate cancer[tiab]) AND (microRNA*[tiab] OR 
microRNA \& mRNA expression profile

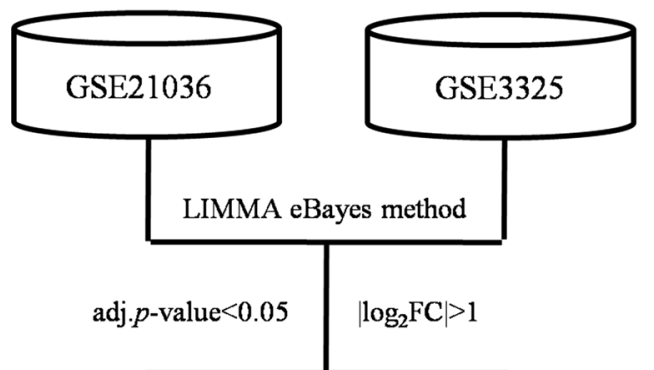

DE-microRNAs

DE-mRNAs
microRNA-mRNA regulatory pairs
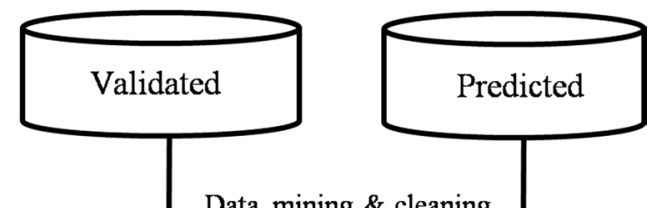

Data mining \& cleaning

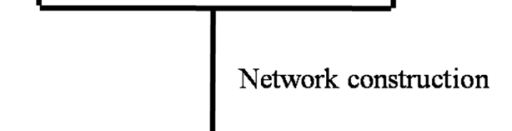

Human microRNA-mRNA regulatory network

Mapping \& Extracting

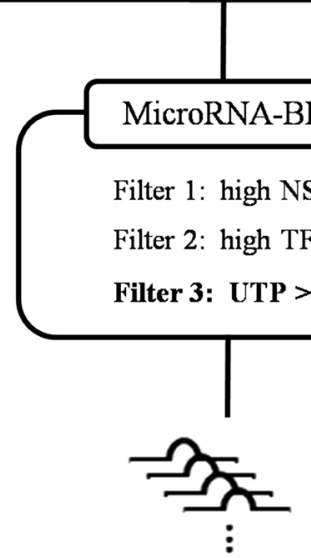

Candidate microRNA biomarkers

Validation

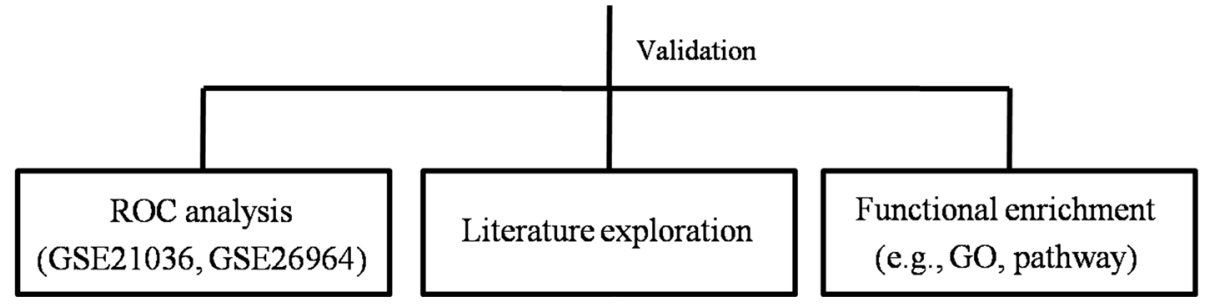

Fig. 1 The schematic pipeline for MPCa microRNA biomarker identification. LIMMA linear models for microarray data analysis; eBayes the empirical bayes; adj.p value adjusted $p$ value; FC fold change; DE differentially expressed; MicroRNA-BD microRNA biomarker discovery; NSR number of single-line regulation; TFP transcription factor gene percentage; UTP percentage of transcription factor genes in microRNA unique targets; $R O C$ receiver operating characteristic curve; GO Gene Ontology; MPCa metastatic prostate cancer

Table 1 Summary of the microRNA and mRNA dataset used in this study

\begin{tabular}{|c|c|c|c|c|c|}
\hline Category & RNA type & GEO accession & Platform & Sample source & $\begin{array}{l}\text { Number } \\
\text { of samples } \\
\text { (PPCa/MPCa) }\end{array}$ \\
\hline \multirow[t]{2}{*}{ Prediction } & microRNA & GSE21036 & GPL8227 & Prostate tissue & $113(99 / 14)$ \\
\hline & mRNA & GSE3325 & GPL570 & Prostate tissue & $9(5 / 4)$ \\
\hline Validation & microRNA & GSE26964 & GPL8469 & Prostate tissue & $13(6 / 7)$ \\
\hline
\end{tabular}

PPCa primary prostate cancer; MPCa metastatic prostate cancer 
miRNA*[tiab]) AND (biomarker*[tiab] OR marker*[tiab] OR indicator*[tiab] OR predict*[tiab])".

\section{Differentially expressed microRNAs (DE-microRNAs) and mRNAs (DE-mRNAs) extraction}

The DE-microRNAs and DE-mRNAs were extracted based on the comparison of their expressions between $\mathrm{PPCa}$ and MPCa samples using the empirical bayes (eBayes) method in linear models for microarray data analysis (LIMMA) R package [28, 29]. The BenjaminiHochberg false discovery rate method was applied to adjust raw $p$ values [30]. For the gene that is related to multiple probes, we assigned it to the probe that had the most significant variation across its expression profile. The adjusted $p$ value (adj. $p$ value) $<0.05$ and $\mid \log _{2}$ foldchange $\mid>1$ were chosen as the cut-off.

\section{MPCa-specific microRNA-mRNA network construction}

The MPCa-specific microRNA-mRNA network was constructed in two steps: First, a human microRNAmRNA network (termed as the reference network) was built based on both experimentally validated and computationally predicted microRNA-mRNA regulatory data. Compared with our previous work [20,21], the microRNA-mRNA pairs were updated and moreover, the latest nomenclature of microRNAs in miRBase (Release 21) [31] was fully considered before network reconstruction. Here the experimental data were mined from miRTarBase (version 4.5) [32], TarBase (version 6.0) [33], miRecords (version 4.0) [34], and miR2Disease [35] whereas the predicted data included information from HOCTAR (version 2.0) [36], ExprTargetDB [37], and starBase (version 2.0) [38]. To reduce the false positive rate, microRNA-mRNA pairs validated by low-throughput experiments, e.g, real-time PCR etc. were considered in this study while the predicted pairs were selected only when they existed at least in two of the three computational prediction databases. In the second step, the DE-microRNAs and DE-mRNAs were mapped onto the reference to extract the MPCa-specific microRNAmRNA network.

\section{Biomarker microRNA identification based on network vulnerability analysis}

As illustrated in Fig. 2, the microRNA-mRNA relationship can be classified into four types based on their regulatory modes. The POMA model proposed in our previous studies pays more attention to the single-line regulatory power of microRNAs and focuses on the biological functions of their targets [20,21]. After analyzing the sub-structure of microRNA-mRNA network, we found that microRNAs still held the potential to uniquely regulate genes with crucial functions, e.g., transcription factor (TF) genes. As an example, the TF gene G_10 in Fig. 2 is uniquely regulated by $M_{-} 4$. The alteration of single-line regulation is not compensated, and TFs are often key players in many important biological processes, thus it is reasonable to assume that the dysregulation of such regulatory patterns is more likely to alter the system stability and eventually cause the systematic disorder. To strengthen the importance of microRNA regulation on TF genes, we here applied a novel parameter called the unique-regulated TF gene percentage (UTP). Numerically, it is equivalent to the percentage of TF genes in the microRNA unique targets. Finally, our previous model POMA was improved as MicroRNA-BD to identify microRNA biomarkers with the following three measurements for network vulnerability characterization.

Step 1: NSR (number of single-line regulation) measurement is the number of genes that are uniquely regulated by a single microRNA.

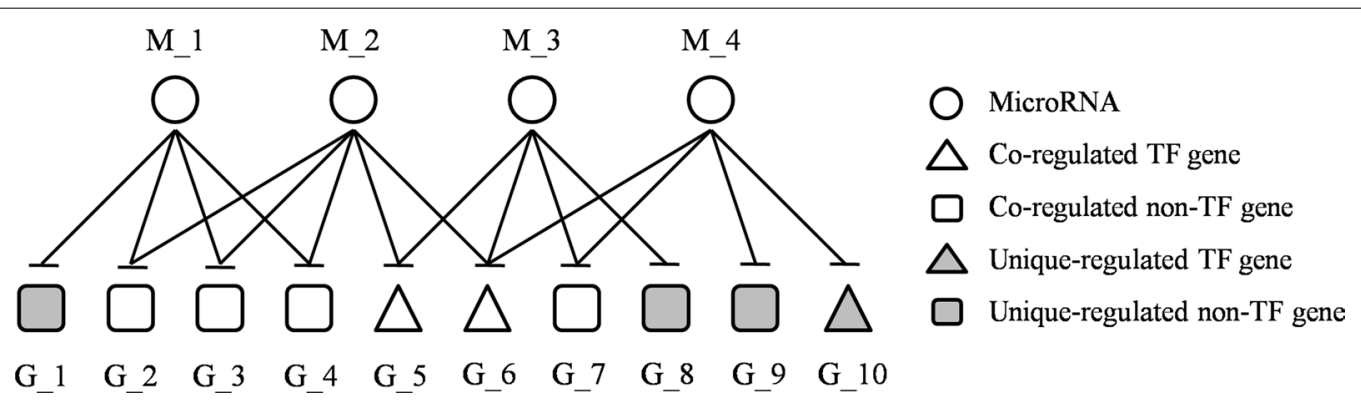

Fig. 2 Schematic description of microRNA-mRNA regulatory types. Four types were defined here, i.e., TF or non-TF genes regulated by multiple or single microRNAs. For example, G_ 1 was uniquely regulated by $M_{-} 1$ whereas TF gene $G \_5$ was co-regulated by $M \_2$ and $M \_3$. The co-regulatory sites are robust since one of the regulations altered can be compensated by others. Here the unique regulatory sites, i.e., single-line regulations, are considered as the vulnerable structure in the network. Meanwhile, microRNAs that target more TF genes seem to be functionally important. $M$ microRNA; G gene; TF transcription factor 
MicroRNAs with significantly high NSR values ( $\mathrm{p}$ value $<0.05$, Wilcoxon signed-rank test) were extracted based on the analysis of MPCaspecific microRNA-mRNA network

Step 2: TFP (transcription factor gene percentage) measurement is the percentage of TF genes targeted by a single microRNA. MicroRNAs with significantly high TFP values ( $p$ value $<0.05$, Wilcoxon signed-rank test) were selected from those screened in Step 1;

Step 3: UTP (unique-regulated TF gene percentage) measurement is defined as the percentage of TF genes in microRNA unique targets. MicroRNAs with UTP $>0$ in Step 2 were identified as candidate biomarkers.

\section{Performance evaluation}

We performed the receiver-operating characteristic (ROC) analysis on both prediction and validation microRNA datasets to evaluate the performance of identified microRNA biomarkers for classifying MPCa and PPCa. The ROC curve and the area under curve (AUC) were drawn and calculated for each of the identified microRNAs using the R package 'epicalc' [39]. The percentage of the reported MPCa microRNA biomarkers in the predicted set was defined as prediction precision to quantify the performance of the model.

\section{Functional enrichment analyses}

To validate the association between the targets of candidate microRNA biomarkers and $\mathrm{PCa}$ metastasis, we performed Gene Ontology (GO) annotation, Kyoto Encyclopedia of Genes and Genomes (KEGG) [40] pathway analyses and Ingenuity Pathway Analysis (IPA) [41] using Database for Annotation, Visualization and Integrated Discovery (DAVID, version 6.7) [42] and IPA program [41], respectively. Here the targets of identified microRNA biomarkers were retrieved from human microRNAmRNA network and Benjamini-Hochberg method was used to adjust raw $p$ values for enrichment analysis. The top ten significantly enriched terms (adj. $p$ value $<0.05$ ) were selected and further studied for their correlations with PCa metastasis through literature validation.

\section{Results}

Biomarker microRNAs for predicting PCa metastasis

A total of 67 literature reported PCa microRNA biomarkers were manually collected (see Additional file 1). The human microRNA-mRNA network included 48,868 regulatory pairs among 618 microRNAs and 9526 genes/
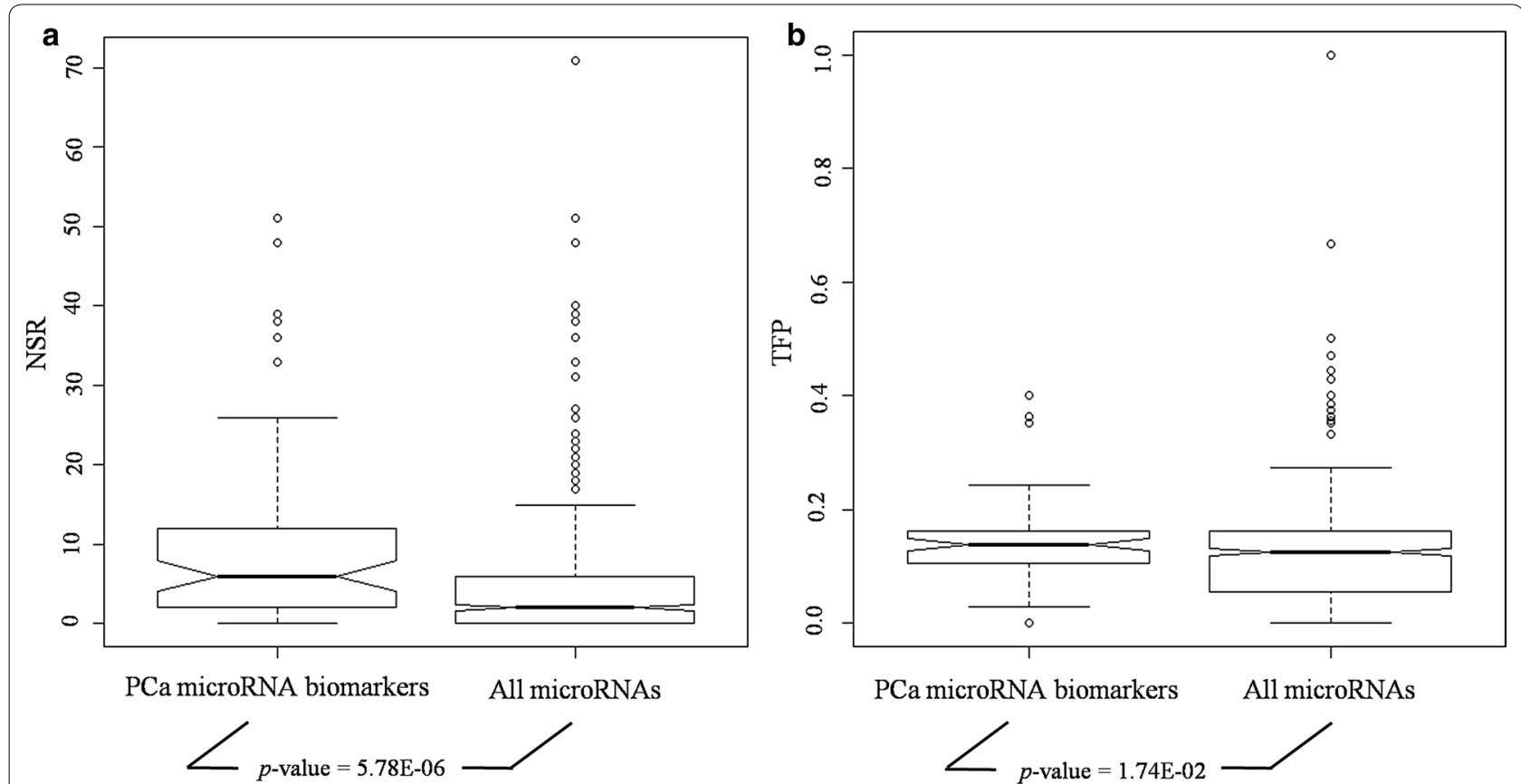

Fig. 3 Topological and functional characterization of reported PCa microRNA biomarkers. a NSR distribution of reported PCa microRNA biomarkers and all microRNAs in human microRNA-mRNA network. $\mathbf{b}$ TFP distribution of reported PCa microRNA biomarkers and all microRNAs in human microRNA-mRNA network. The statistical significance was calculated using Kolmogorov-Smirnov test. NSR number of single-line regulation; TFP transcription factor gene percentage; PCa prostate cancer 
mRNAs. As shown in Fig. 3, Additional files 1 and 2, respectively, $\mathrm{PCa}$ biomarker microRNAs had significant regulatory power in the network, which convinced the priori evidence for biomarker discovery [20, 21]. Moreover, more than $53.7 \%(36 / 67)$ of these microRNAs had $\mathrm{UTP}>0$, which was approximately twice greater than that in the whole network (27.5\%, 170/618).

Based on the selected sample data, 91 DE-microRNAs and 970 DE-mRNAs were statistically identified. The MPCa-specific microRNA-mRNA network contained 505 microRNA-mRNA regulatory pairs among 65 microRNAs and 263 mRNAs (see Additional file 3). Seven microRNAs, i.e., miR-204-5p, miR-101-3p, miR145-5p, miR-198, miR-152, miR-130a-3p and miR-363-3p tended to have significantly high NSR and TFP values in MPCa-specific microRNA-mRNA network (see Additional file 4). The UTP measurements for miR-130a-3p and miR-363-3p are 0 , therefore, the remaining five microRNAs, i.e., miR-204-5p, miR-101-3p, miR-145-5p, miR-198, and miR-152 were screened as putative biomarkers for predicting PCa metastasis after MicroRNABD filtration.

As shown in Table 2, four of the identified microRNAs, i.e., miR-204-5p, miR-101-3p, miR-145-5p, and miR152 , were significantly down-regulated in $\mathrm{MPCa}$ group whereas miR-198, was over-expressed in MPCa samples compared with PPCa. The ROC curves for their PCa metastasis prediction performance were shown in Fig. 4. In the prediction set GSE21036 and another independent validation set GSE26964, the AUC ranged from 0.70 to 0.99 and from 0.71 to 0.93 , respectively. Overall, miR145-5p, miR-204-5p, and miR-152 achieved the best performance (AUC>0.80) on PPCa and MPCa subtyping, and the AUC distribution of the five microRNAs in two datasets was highly consistent, which indicated the predictive power of the identified microRNA biomarkers for discriminating between MPCa and PPCa.

\section{Literature validation of the identified microRNA biomarkers}

All of the five microRNAs were involved in PCa pathogenesis according to PubMed literature reports. In particular, two microRNAs (40\%, 2/5), i.e., miR-145-5p and miR-101-3p, have been reported as potential biomarkers for human PCa metastasis [27, 43, 44]. As a tumor suppressor microRNA, the down-regulation of miR145 (namely miR-145-5p) could cause cell invasion and migration in PCa progression [27]. Huang et al. showed that miR-145 regulated the characteristics of cancer stem cells and played important roles in the progression of $\mathrm{PCa}$ bone metastasis [45]. Moreover, miR-145 is a direct target of $\mathrm{p} 53$, and the loss of wild-type p53 could promote PCa bone metastasis by partially repressing miR-145 expression [46]. Chakravarthi et al. showed that the loss of miR-101 (namely miR-101-3p) may affect the expression of SUB1 and lead to the activation of known oncogenes driving PCa metastasis [47]. Besides, miR-204-5p and miR-152 were also associated with PCa progression and metastasis. Lin et al. [48] found that miR-204-5p was a tumor suppressor and could promote apoptosis through regulating BCL2 in PCa cells. Todorova et al. studied the effect of miR-204 (namely miR-204-5p) modulation on important TFs for PCa bone marrow metastasis and uncovered that this microRNA was dysregulated in MPCa in vitro [49]. Theodore et al. analyzed the microRNA expression profile data and found the ethnic difference of miR-152 expression between African American (AA) and Caucasian PCa patients. On the other hand, the epigenetic regulation of miR-152 and DNMT1 may contribute to the aggressiveness of $\mathrm{PCa}$ tumors, especially to AA PCa patients [50]. Last but not least, miR198 was found to be up-regulated in high grade (Gleason score $\geq 8$ ) PCa tumors, which would help recognize the aggressive behavior of PCa [51]. However, the filtered two microRNAs, i.e., miR-130a-3p and miR-363-3p, did not show any reliable correlation with PCa metastasis so far. In summary, MicroRNA-BD outperformed previous models and improved the microRNA biomarker

Table 2 Details for the identified microRNA biomarkers

\begin{tabular}{|c|c|c|c|c|c|c|}
\hline microRNA ID & $\begin{array}{l}\text { Adj.p value (PPCa vs } \\
\text { MPCa) }\end{array}$ & $\log _{2}(F C)$ & Target number & NSR & TFP & UTP \\
\hline miR-204-5p & $3.43 \mathrm{E}-08$ & -2.0896 & 21 & 13 & 0.2857 & 0.3846 \\
\hline miR-101-3p & $1.26 \mathrm{E}-08$ & -1.0684 & 24 & 3 & 0.2917 & 0.3333 \\
\hline miR-145-5p & $8.00 E-25$ & -3.2157 & 12 & 3 & 0.2500 & 0.3333 \\
\hline miR-198 & 7.67E-05 & 1.2564 & 12 & 5 & 0.3333 & 0.2000 \\
\hline miR-152 & $3.67 \mathrm{E}-08$ & -1.0146 & 17 & 6 & 0.2941 & 0.1667 \\
\hline
\end{tabular}

PPCa primary prostate cancer; MPCa metastatic prostate cancer; adj. $p$ value adjusted $p$ value; FC fold change; NSR number of single-line regulation; TFP transcription factor gene percentage; UTP percentage of transcription factor genes in microRNA unique targets 

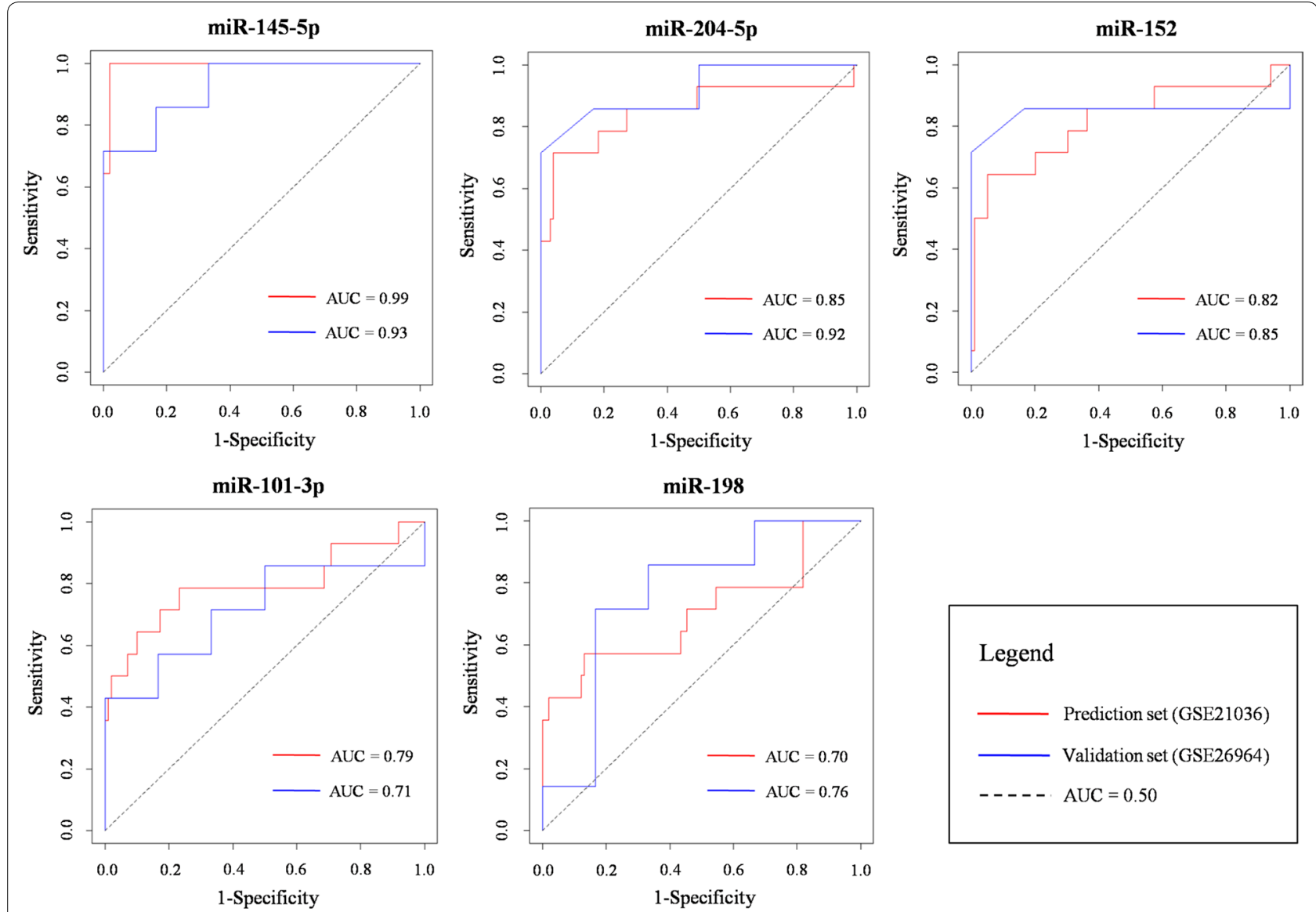

Fig. 4 ROC analysis for the identified microRNA biomarkers. The AUC distribution in the prediction set GSE21036 and another independent validation set GSE26964 ranged from 0.70 to 0.99 and from 0.71 to 0.93 , respectively. Red curve: GSE21036; blue curve: GSE26964. PPCa primary prostate cancer; MPCa metastatic prostate cancer; $R O C$ receiver operating characteristic curve; AUC area under the curve

prediction precision from $28.6 \%(2 / 7)$ to $40 \%(2 / 5)$ in this case study.

We further evaluated the relationship between the targets of identified microRNA biomarkers and PCa metastasis. As illustrated in Fig. 5, the TF gene STAT1, which was uniquely regulated by miR-145-5p in the $\mathrm{MPCa}-$ specific microRNA-mRNA network, could be activated by Endoglin. In the study, Endoglin was shown to suppress the cell invasion of $\mathrm{PCa}$ and inhibit PCa metastasis [52]. Meanwhile, several uniquely-regulated non-TF genes, such as DDR2 and MRC2, also participated in the PCa metastatic progression [53, 54]. For instance, DDR2 regulated the promoter activity of parathyroid hormonerelated protein and thereby facilitated the bone metastasis of PCa [53]. In addition, EMP1 suppressed PCa cell proliferation and invasion by regulating caspase- 9 and VEGFC protein [55], and it was co-regulated by miR101-3p, miR-204-5p and miR-152 in the network. Some other co-regulated genes, including PTEN and MYC, were also functional during PCa evolution [56, 57].

\section{Gene ontology (GO) annotation}

The GO analysis was performed using the online tool DAVID at three levels, i.e., biological process (BP), cellular component (CC), and molecular function (MF). The statistically significant terms (adj.p value $<0.05$ ) of each level were summarized in Additional file 5 . Here we mainly focused on the top ten significantly enriched terms for in-depth analyses. As shown in Table 3, at the BP level, the most significant terms were closely relevant to cell cycle, metabolic processes and cell death. Accumulating evidence demonstrated that a number of genes as well as non-coding RNAs played functions in PCa metastasis by dramatically activating or inhibiting the cell cycle process [58-60]. Wang et al. analyzed the gene expression data of castration-resistant $\mathrm{PCa}$ and found that the identified regulatory modules were also enriched in the phosphorus metabolic process [61]. At the CC level, the enriched terms were mainly concentrated on nuclear lumen, nucleoplasm, and organelle lumen. Nucleophosmin (NPM1) is a nucleoprotein and associated with 


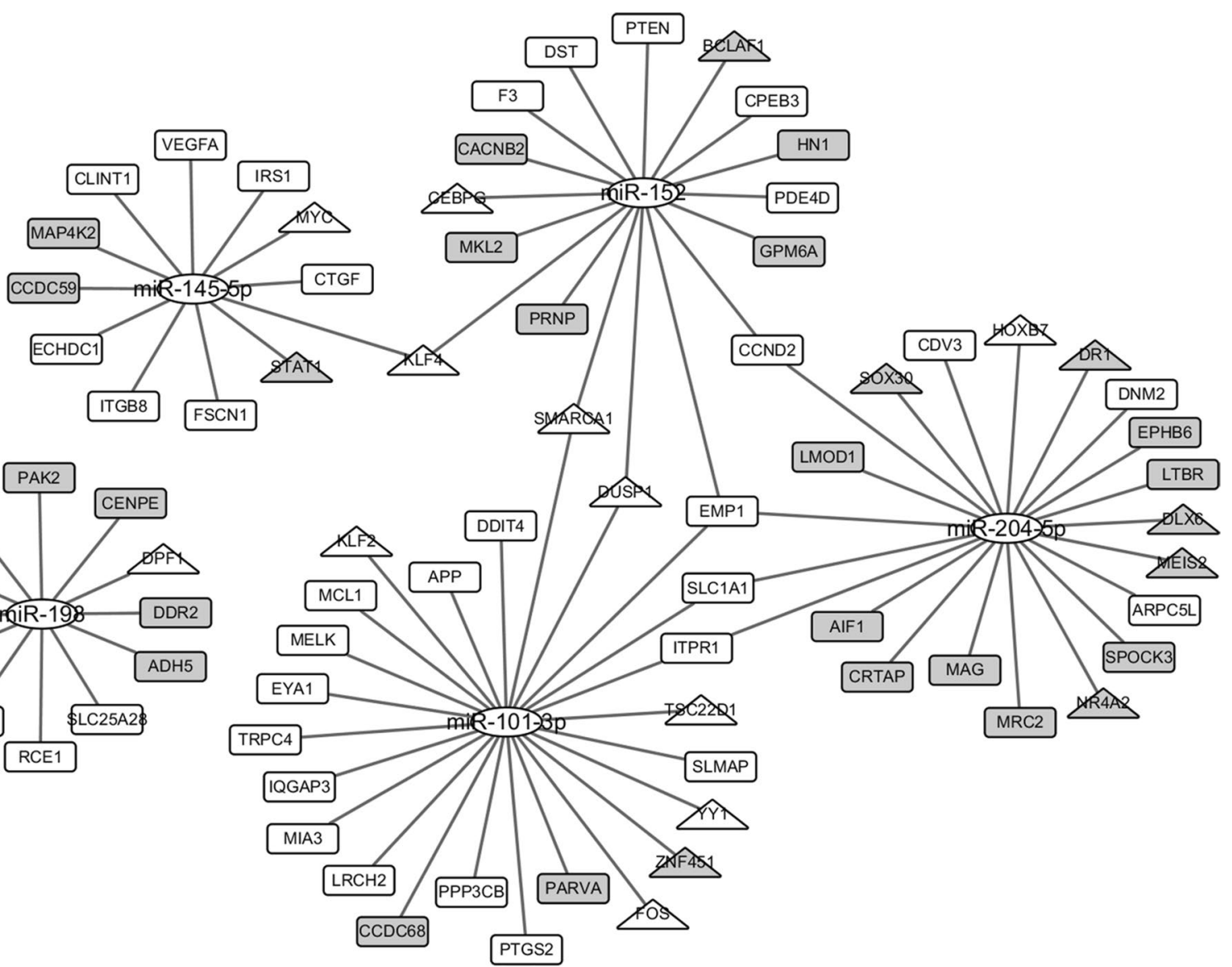

Fig. 5 Identified biomarker microRNAs and their targets in MPCa-specific microRNA-mRNA network. Elliptic, triangular and rectangular nodes represent microRNAs, TF genes and non-TF genes, respectively. Nodes in grey represent genes that are uniquely regulated by single microRNAs in the network. MPCa metastatic prostate cancer; TF transcription factor

tumor growth. Destouches et al. showed that the phosphorylated NPM1 may interact with androgen receptor in nucleoplasm, which is biologically important in $\mathrm{PCa}$ progression [62]. At the MF level, most enriched terms were linked with molecular activities, including transcription regulator activity, transcription repressor activity, transcription activator activity, etc. Grubb RL et al. found that the transcription regulatory protein STAT3 differed statistically in PCa with high Gleason grade $(\geq 8)$ [63]. Xiao et al. showed that the loss expression of PLZF, a transcription repressor in oncogenesis, correlated with PCa tumor aggressiveness [64], which highlighted the functional importance of transcription repressor activity in PCa metastasis.

\section{Pathway enrichment analysis}

To investigate the functional mechanisms of the five microRNA candidates, we performed the KEGG and IPA pathway enrichment analysis on their targets using DAVID and IPA program, respectively. The significantly enriched terms (adj. $p$ value $<0.05$ ) were listed in Additional files 6 and 7, respectively. Here the top ten significant terms were mainly selected for further literature exploration. As shown in Fig. 6a, the most meaningful KEGG terms were Axon guidance, Pathways in cancer, Cell cycle, Prostate cancer, and MAPK signaling pathway. Ding et al. found that Semaphorin 4F (S4F), which played important roles in embryologic axon guidance, was a key regulator in the tumor microenvironment and a biomarker of aggressive PCa [65]. McNair et al. uncovered that the cell cycle-coupled expansion of AR activity promoted the progression of $\mathrm{PCa}$ and was related to the development of PCa metastases [66]. As shown in Fig. 7, the targets enriched in the prostate cancer pathway had close relations with cell cycle. They potentially mediated the process of cell proliferation and cell survival. Two tumor suppressors, i.e., PTEN and p27 (CDKN1B), which showed the prognostic or therapeutic value in $\mathrm{PCa}$ metastasis and recurrence [56, 67], were functionally regulated by the identified microRNAs. 
Table 3 Top ten significant GO terms enriched by targets of the identified microRNA biomarkers

\begin{tabular}{|c|c|c|c|}
\hline Category & GO terms & Number of enriched genes & Adj. $p$ value \\
\hline \multirow[t]{10}{*}{$\mathrm{BP}$} & Mitotic cell cycle & 60 & $3.52 \mathrm{E}-07$ \\
\hline & Phosphorus metabolic process & 112 & $4.00 \mathrm{E}-06$ \\
\hline & Phosphate metabolic process & 112 & 4.00E-06 \\
\hline & Regulation of apoptosis & 97 & $3.51 \mathrm{E}-06$ \\
\hline & Positive regulation of cell proliferation & 61 & $2.83 \mathrm{E}-06$ \\
\hline & Cell cycle process & 75 & $3.16 \mathrm{E}-06$ \\
\hline & Regulation of programmed cell death & 97 & $2.92 \mathrm{E}-06$ \\
\hline & Regulation of cell death & 97 & $3.02 \mathrm{E}-06$ \\
\hline & Cell cycle & 93 & $4.11 \mathrm{E}-06$ \\
\hline & Regulation of transcription from RNA polymerase II promoter & 88 & $6.10 E-06$ \\
\hline \multirow[t]{10}{*}{ CC } & Nuclear lumen & 162 & 1.17E-13 \\
\hline & Nucleoplasm & 110 & $7.25 \mathrm{E}-12$ \\
\hline & Organelle lumen & 182 & $1.05 \mathrm{E}-11$ \\
\hline & Membrane-enclosed lumen & 183 & $2.45 \mathrm{E}-11$ \\
\hline & Intracellular organelle lumen & 176 & $5.37 \mathrm{E}-11$ \\
\hline & Nucleoplasm part & 77 & $1.95 \mathrm{E}-10$ \\
\hline & Intracellular non-membrane-bounded organelle & 212 & $5.33 \mathrm{E}-06$ \\
\hline & Non-membrane-bounded organelle & 212 & 5.33E-06 \\
\hline & Chromatin remodeling complex & 18 & $3.26 \mathrm{E}-05$ \\
\hline & Nucleolus & 72 & $1.81 \mathrm{E}-04$ \\
\hline \multirow[t]{10}{*}{ MF } & Transcription regulator activity & 163 & $1.16 \mathrm{E}-09$ \\
\hline & Transcription repressor activity & 50 & $1.35 \mathrm{E}-06$ \\
\hline & Transcription factor binding & 67 & 4.45E-06 \\
\hline & Transcription activator activity & 56 & $1.36 \mathrm{E}-05$ \\
\hline & Transcription factor activity & 100 & $1.41 \mathrm{E}-04$ \\
\hline & Protein kinase activity & 68 & $5.01 \mathrm{E}-04$ \\
\hline & Transcription cofactor activity & 46 & $1.07 \mathrm{E}-03$ \\
\hline & Protein serine/threonine kinase activity & 50 & 3.67E-03 \\
\hline & DNA binding & 190 & $5.00 E-03$ \\
\hline & Phosphoprotein phosphatase activity & 25 & $8.40 \mathrm{E}-03$ \\
\hline
\end{tabular}

GO gene ontology; BP biological process; CC cellular component; $M F$ molecular function; adj. $p$ value: adjusted $p$ value

As shown in Fig. 6b, several pathways were also significantly enriched in IPA, such as molecular mechanisms of cancer, axonal guidance signaling, and ERK/MAPK signaling etc. Substantial efforts have convinced that the MAPK signaling was involved in the progression of advanced or metastatic PCa [68-70]. As illustrated in Fig. 8, the targets of identified biomarkers were almost enriched in the hubs of ERK/MAPK signaling, which demonstrated the regulatory power of these microRNAs. Chen et al. reviewed the relation between PI3K/ AKT signaling and $\mathrm{PCa}$ tumorigenesis and pointed out that this pathway regulated tumor cell invasion during the metastasis of PCa cells [71]. Kassi et al. reported that glucocorticoids could mediate the transcriptional regulation of genes which were functional in PCa cell growth, inflammation, differentiation, apoptosis, and metastasis, and the glucocorticoids receptor signaling participated in $\mathrm{PCa}$ through cross talking with other signaling cascades [72]. Another well-studied pathway associated with $\mathrm{PCa}$ development is TGF- $\beta$ signaling. It is reported that the TGF- $\beta$ pathway held the potential to maintain tissue homeostasis and was functional during cancer cell proliferation [73]. Bonci et al. found that the concomitant decrease of miR-15/16 and increase of miR-21 could abnormally activate TGF- $\beta$ signaling, leading to the invasion, migration and distant bone metastasis of PCa cells [12]. Zhang et al. demonstrated that the TGF- $\beta$ pathway was significantly enriched by genes in the identified biomarker modules for $\mathrm{PCa}$ subtyping [19]. In this study, as illustrated in Additional files 7 and 8 , respectively, the TGF- $\beta$ signaling was also shown statistical significance as the target of the five microRNAs. Moreover, TGFBR1 and TGFBR2, two transforming growth factor beta receptors with 


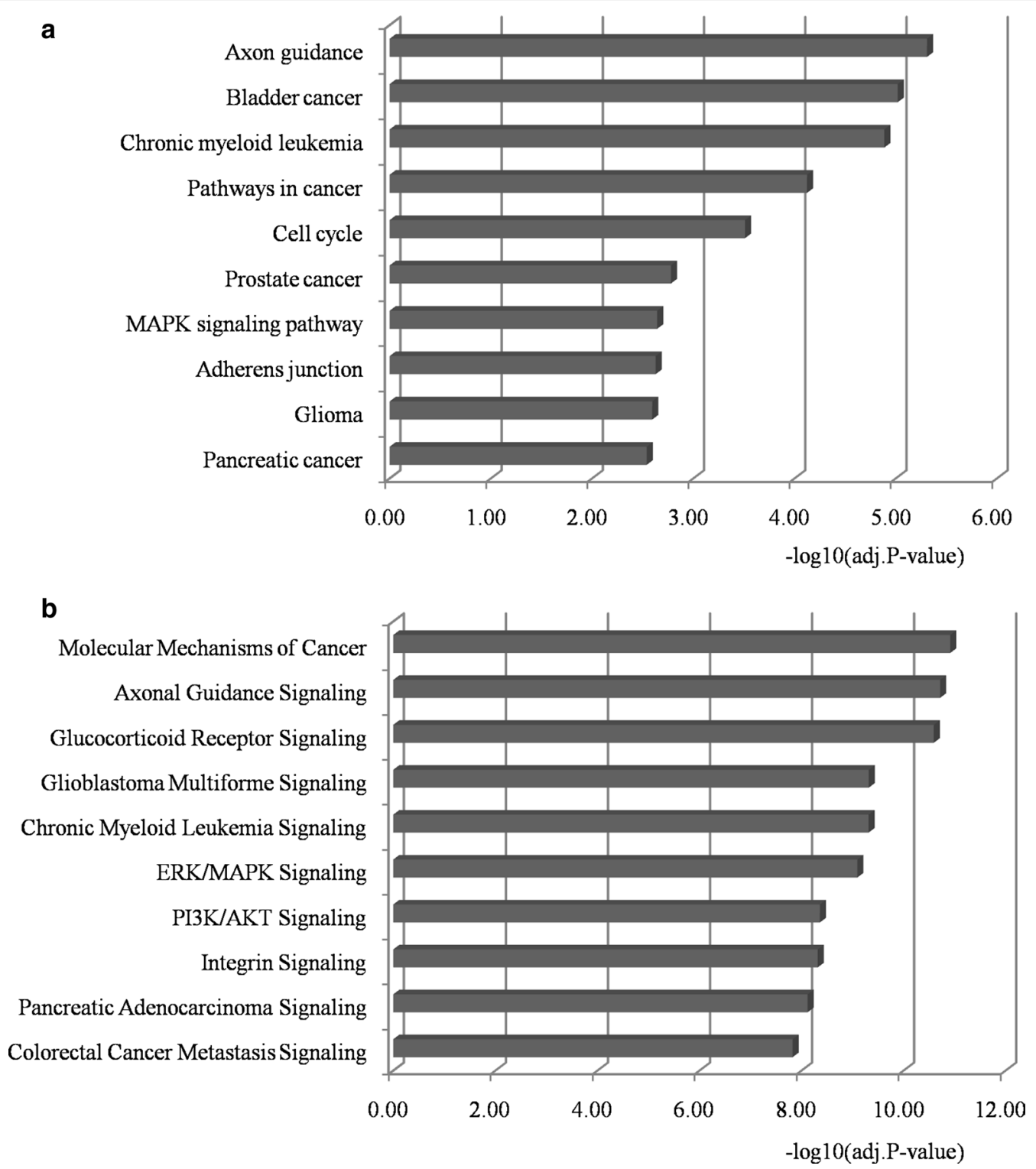

Fig. 6 Pathway enrichment analysis for targets of the identified microRNA biomarkers. The statistical significance level (adj. $p$ value) was negative 10-based log transformed. a The top ten significant KEGG terms. b The top ten significant IPA terms. adj.p value adjusted $p$ value; KEGG Kyoto Encyclopedia of Genes and Genomes; IPA ingenuity pathway analysis

the power of transferring TGF-beta signal from the extracellular space to the cytoplasm, were closely regulated by these microRNAs, which could strengthen the importance of our findings.

We noticed that the targets of the identified microRNAs were also correlated with bladder cancer, glioma, pancreatic cancer and colorectal cancer, which indicated the similarity of pathogenesis between PCa metastasis and other cancers. For example, TRAP1/Hsp75 has been reported to be a molecular marker in metastatic $\mathrm{PCa} . \mathrm{Li}$ et al. proved that the expression of TRAP1 was increased in glioma compared with its normal controls, and it could be a useful prognostic factor in glioma management [74]. The RNASEL germline variants were associated with not only familial $\mathrm{PCa}$, but also pancreatic cancer, which indicated the potential mechanisms between pancreatic cancer and PCa development [75].

\section{Discussion}

$\mathrm{PCa}$ is a commonly diagnosed cancer among males around the world. While the overall survival rate has increased these years, the metastasis is still a leading 


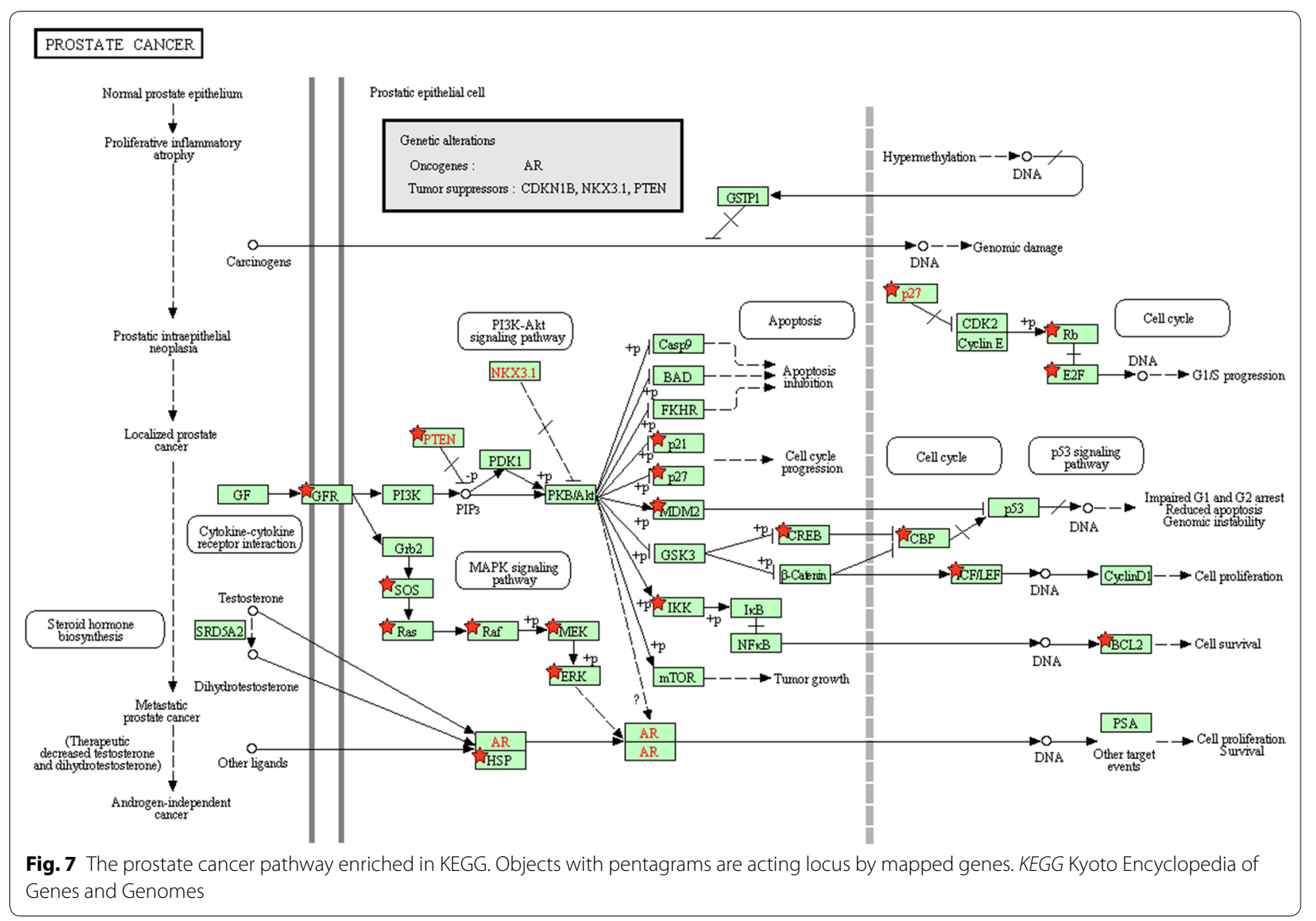

cause of PCa death [76]. The early detection of metastatic signature is important for monitoring PCa prognosis and helping design personalized therapeutic strategies. It is widely acknowledged that microRNAs are a class of functional regulators in biological processes and have good sensitivity and specificity to be biomarkers for disease initiation and progression [77].

In this study, we integrated microRNA/mRNA expression data with network structural knowledge and improved the bioinformatics model to screen candidate microRNA biomarkers for predicting PCa metastasis. Compared with the previous work, we updated the reference microRNA-mRNA network by carefully integrating recently reported human microRNA-mRNA associations based on microRNA nomenclature. Besides the single-line regulatory power (NSR) and biological roles of microRNAs (TFP), we considered the functional importance of genes that are uniquely regulated by single microRNAs in this model. It is reasonable that the singleline regulatory site in the network is relatively vulnerable and TFs are important regulators in various biological activities. The incorporative analysis of structural and functional characteristics in microRNA-mRNA regulation would strengthen the evalutaion of microRNA regulatory power. Hence we defined a new parameter called UTP to quantify the special regulation of given microRNAs, that is, the percentage of TF genes in microRNA unique targets.

Based on this computational model, a total of five microRNAs, i.e., miR-204-5p, miR-101-3p, miR-145-5p, miR-198, and miR-152, were identified as candidate biomarkers for PCa metastasis prediction. Among them, miR-145-5p and miR-204-5p were validated as tumor suppressor microRNAs, and their down-regulation could disorder cell cycle processes and finally result in $\mathrm{PCa}$ invasion [27, 48]. More importantly, miR-145-5p and miR-101-3p have been reported as biomarkers for evaluating PCa metastasis previously [43, 44]. The remaining three were also confirmed to be connected with the development of metastatic or high-grade PCa according to PubMed literature searches. Compared with POMA solely using NSR and TFP as filters, the MicroRNA-BD model improved the prediction precision from $28.6 \%$ $(2 / 7)$ to $40 \%(2 / 5)$ in this case study. Furthermore, the 


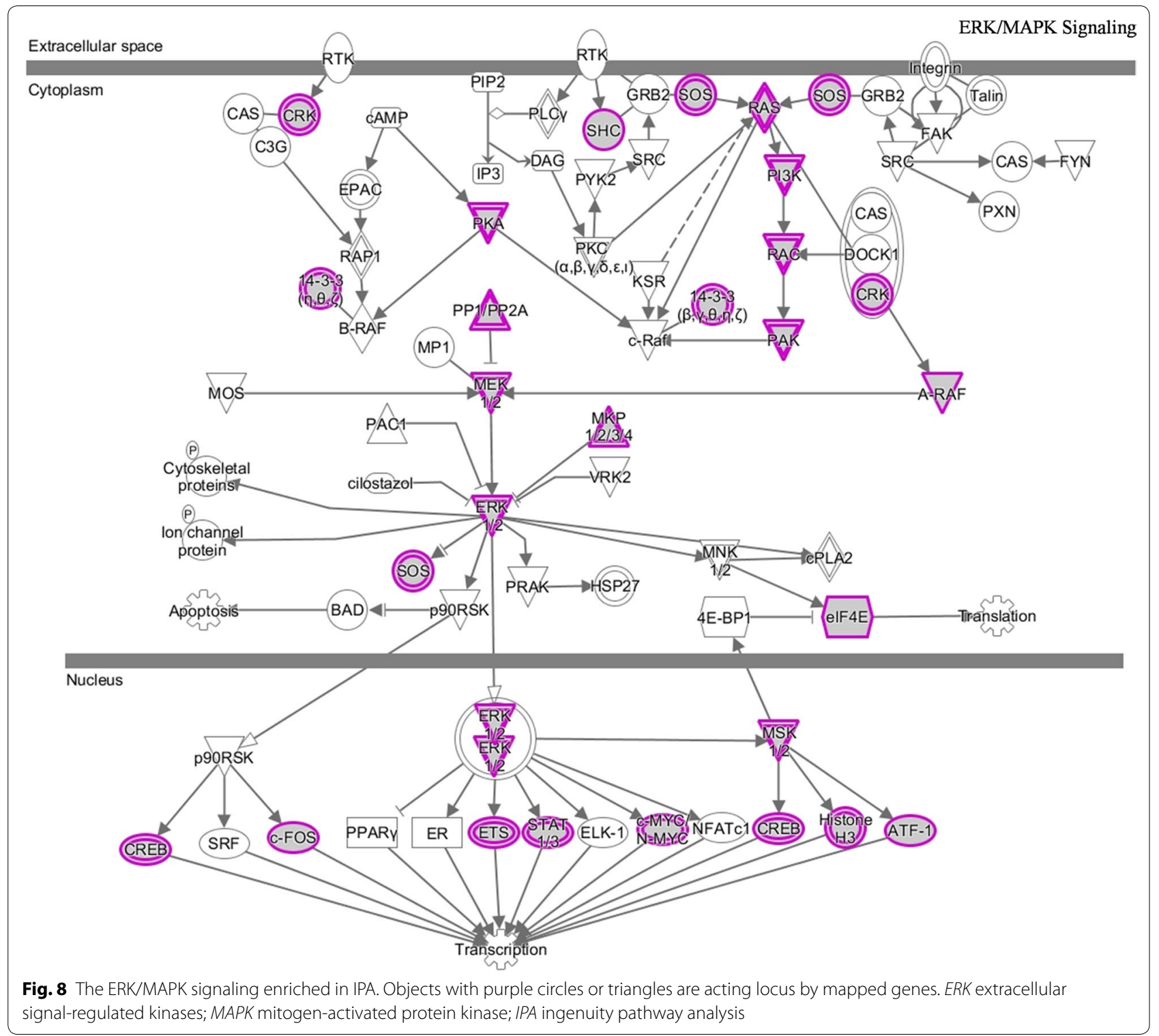

AUC values for the prediction performance of the five microRNAs, respectively, ranged from 0.70 to 0.99 and from 0.71 to 0.99 in two datasets, i.e., the prediction set and another independent validation set, which indicated the power of the identified biomarkers for PCa prognosis and metastasis tracking.

We further investigated the pathogenic mechanisms of the identified microRNAs in PCa metastasis through GO and pathway enrichment analyses. The cell cycle process, for example, is one of the most significant terms enriched in GO and KEGG pathway, which supported the pivotal view of cell cycle-mediated PCa carcinogenesis $[66,78,79]$. The prostate cancer pathway, ERK/MAPK signaling, and TGF- $\beta$ signaling are well-studied in PCa metastasis, and we found that most of the targets of identified microRNA biomarkers were the key components of these pathways, meanwhile, some tumor suppressor genes, e.g., PTEN and p27, were regulated by the identified microRNAs. In addition, the remaining pathways, such as axonal guidance [65], PI3K/AKT signaling [71], glucocorticoids receptor signaling [72], pancreatic cancer [75], and molecular mechanisms of cancer [80] etc., were all involved in PCa progression and metastasis according to previous reports.

Compared with the existing approaches for $\mathrm{PCa}$ metastasis investigation, our model detects microRNA biomarkers based on statistical evidences from a combination of network sub-structural and functional 
analyses. In the study of Zhang et al., five microRNAmRNA modules were identified for PPCa and MPCa respectively based on the correction and clustering analysis on microRNA and mRNA datasets [19]. Similar to this idea, our study also utilized microRNAmRNA network information. However, the network in our model was concentrated more as a biological system, where special regulatory patterns altering its stability were concluded as the principle for biomarker prediction. Another major difference between the two approaches regards to the methodology for sub-network extraction. In contrast to the two types of network modules, i.e., PPCa- and MPCa-module, built in Zhang et al. using clustering and condensing techniques, [19], our model constructed microRNAmRNA network specific to $\mathrm{PCa}$ metastasis in order to capture the changing signatures during $\mathrm{PCa}$ evolution, and only single microRNAs could be screened as candidate biomarkers for PCa subtyping. Considering the results, the miR-145-5p detected by our model was also involved in one of the modules in Zhang et al. [19], which convinced the underlying power of this microRNA for PCa metastasis predicting. From pathway angles, both Zhang et al. [19] and Bonci et al. [12] demonstrated the importance of TGF- $\beta$ signaling as microRNA targets in PCa progression and metastasis. As described in the section of 'Pathway enrichment analysis', we found that genes regulated by the five biomarker microRNAs were similarly enriched in TGF- $\beta$ signaling, which indicated the pathway-level consistency of results for PCa carcinogenesis decoding across these studies. Due to the complexity and diversity in PCa development, biomarker microRNAs identified by different methods tended to be highly heterogeneous. In systems biology viewpoints, living organisms are often treated as a holistic framework, thus identifying module or network biomarkers catering to the dynamical nature of PCa pathogenesis for personalized prognosis and treatment is our next-step action.

It should not be ignored that some limitations still existed in this study. Firstly, genes in the present model were treated equally. As we known, the importance of genes in different biological activities is not the same, more functional annotations need to be weighted reasonably. Secondly, only TF genes were selected in the study, the specific knowledge to PCa metastasis could be considered in order to provide precise strategies for MPCa early detection and treatment. Thirdly, only 618 microRNAs were recorded in our reconstructed human microRNA-mRNA network, the network scale should keep pace with the development of newly identified microRNA-mRNA associations. Last but the most important, we are trying to collect human $\mathrm{PPCa}$ and MPCa samples to further perform wet lab verifications for future carcinogenic exploration and translational application.

\section{Conclusion}

In this study, a total of five microRNAs, i.e, miR-204-5p, miR-101-3p, miR-145-5p, miR-198, and miR-152, were identified as candidate biomarkers for predicting $\mathrm{PCa}$ metastasis based on a novel bioinformatics model. The prediction performance, literature exploration and functional enrichment analysis convinced our findings. More clinical validations are needed in our future translational application.

\section{Additional files}

Additional file 1. Literature-reported PCa microRNA biomarkers.

Additional file 2. The NSR, TFP and UTP values for microRNAs in the reconstructed human microRNA-mRNA network.

Additional file 3. MPCa-specific microRNA-mRNA network.

Additional file 4. MicroRNAs with significantly high NSR and TFP values in MPCa-specific microRNA-mRNA network. The $p$ values were calculated using Wilcoxon signed-rank test.

Additional file 5. Significant GO terms enriched by targets of the identified microRNA biomarkers. The $p$ values were adjusted using BenjaminiHochberg method.

Additional file 6. Significant KEGG terms enriched by targets of the identified microRNA biomarkers. The $p$ values were adjusted using BenjaminiHochberg method.

Additional file 7. Significant IPA terms enriched by targets of the identified microRNA biomarkers. The $p$ values were adjusted using BenjaminiHochberg method.

Additional file 8. The TGF- $\beta$ signaling enriched in IPA. Objects with purple circles or triangles were acting locus by mapped genes.

\section{Abbreviations}

PCa: prostate cancer; PPCa: primary prostate cancer; MPCa: metastatic prostate cancer; LIMMA: linear models for microarray data analysis; eBayes: the empirical bayes; adj. $p$ value: adjusted $p$ value; FC: fold change; DE: differentially expressed; TF: transcription factor; MicroRNA-BD: microRNA biomarker discovery; POMA: pipeline of outlier microRNA analysis; NSR: number of single-line regulation; TFP: transcription factor gene percentage; UTP: unique-regulated TF gene percentage; ROC: receiver operating characteristic curve; AUC: area under the curve; GO: gene ontology; BP: biological process; CC: cellular component; MF: molecular function; KEGG: Kyoto Encyclopedia of Genes and Genomes; DAVID: Database for Annotation, Visualization and Integrated Discovery; IPA: ingenuity pathway analysis.

\section{Authors' contributions}

BS and YL conceptualized the research and developed the bioinformatics model. YL, CD, and ZS collected the data. YL, FC, LS, XT and HD performed the computational analysis. YL, JC and BS drafted and revised the manuscript. All authors read and approved the final manuscript.

\section{Author details}

${ }^{1}$ Center for Systems Biology, Soochow University, Suzhou 215006, China. ${ }^{2}$ Department of Genetics \& Systems Biology Institute, Yale University School of Medicine, West Haven, CT 06516, USA. ${ }^{3}$ Department of Statistics and Data Science, Yale University, New Haven, CT 06511, USA. ${ }^{4}$ Jiangsu Health Vocational College, Nanjing 211800, China. ${ }^{5}$ Suzhou Industrial Park Institute of Services Outsourcing, Suzhou 215123, China. ${ }^{6}$ School 
of Chemistry, Biology and Material Engineering, Suzhou University of Science and Technology, Suzhou 215011, China. ${ }^{7}$ Center for Translational Biomedical Informatics, Guizhou University School of Medicine, Guiyang 550025, China. ${ }^{8}$ Institute for Systems Genetics, West China Hospital, Sichuan University, Chengdu 610041, China.

\section{Acknowledgements}

The authors gratefully thank the academic editor and the anonymous reviewers for their insightful comments and suggestions to improve this manuscript. They also thank Prof. Luonan Chen at Key Laboratory of Systems Biology, Shanghai Institute of Biological Sciences, Chinese Academy of Sciences for providing the IPA analysis.

\section{Competing interests}

The authors delcare that they have no competing interests.

\section{Availability of data and materials}

The data generated or analysed during this study are available from the corresponding author upon reasonable request.

\section{Consent for publication}

Not applicable.

\section{Ethics approval and consent to participate} Not applicable.

\section{Funding}

This study was supported by the National Natural Science Foundation of China (Grant Nos. 31670851, 31470821,31600670, 91530320, and 31770903), National Key Research and Development Program of China (2016YFC1306605).

\section{Publisher's Note}

Springer Nature remains neutral with regard to jurisdictional claims in published maps and institutional affiliations.

\section{Received: 1 March 2018 Accepted: 5 May 2018}

Published online: 21 May 2018

\section{References}

1. Siegel RL, Miller KD, Jemal A. Cancer statistics, 2015. CA Cancer J Clin. 2015:65:5-29.

2. Chen J, Shao P, Cao Q, Li P, Li J, Cai H, Zhu J, Wang M, Zhang Z, Qin C, Yin C. Genetic variations in a PTEN/AKT/mTOR axis and prostate cancer risk in a Chinese population. PLOS ONE. 2012;7:e40817.

3. Fu Q, Liu X, Liu Y, Yang J, Lv G, Dong S. MicroRNA-335 and -543 suppress bone metastasis in prostate cancer via targeting endothelial nitric oxide synthase. Int J Mol Med. 2015;36:1417-25.

4. Bartel DP. MicroRNAs: genomics, biogenesis, mechanism, and function. Cell. 2004;116:281-97.

5. Cui Q, Yu Z, Purisima EO, Wang E. Principles of microRNA regulation of a human cellular signaling network. Mol Syst Biol. 2006;2:46.

6. Dang TM, Wong WC, Ong SM, Li P, Lum J, Chen J, Poidinger M, Zolezzi F, Wong SC. MicroRNA expression profiling of human blood monocyte subsets highlights functional differences. Immunology. 2015:145:404-16.

7. Chen X, Ba Y, Ma L, Cai X, Yin Y, Wang K, Guo J, Zhang Y, Chen J, Guo X, et al. Characterization of microRNAs in serum: a novel class of biomarkers for diagnosis of cancer and other diseases. Cell Res. 2008;18:997-1006.

8. Mitchell PS, Parkin RK, Kroh EM, Fritz BR, Wyman SK, Pogosova-Agadjanyan EL, Peterson A, Noteboom J, O'Briant KC, Allen A, et al. Circulating microRNAs as stable blood-based markers for cancer detection. Proc Natl Acad Sci USA. 2008;105:10513-8.

9. He F, Lv P, Zhao X, Wang X, Ma X, Meng W, Meng X, Dong S. Predictive value of circulating miR-328 and miR-134 for acute myocardial infarction. Mol Cell Biochem. 2014;394:137-44.

10. Wang L, Song G, Tan W, Qi M, Zhang L, Chan J, Yu J, Han J, Han B. MiR-573 inhibits prostate cancer metastasis by regulating epithelial-mesenchymal transition. Oncotarget. 2015:6:35978-90.
11. Damodaran C, Das TP, Papu John AM, Suman S, Kolluru V, Morris TJ, Faber EN, Rai SN, Messer JC, Alatassi H, Ankem MK. miR-301a expression: a prognostic marker for prostate cancer. Urol Oncol. 2016;34(336):e313-20.

12. Bonci D, Coppola V, Patrizii M, Addario A, Cannistraci A, Francescangeli F, Pecci R, Muto G, Collura D, Bedini R, et al. A microRNA code for prostate cancer metastasis. Oncogene. 2016;35:1180-92.

13. Tang Y, Yan W, Chen J, Luo C, Kaipia A, Shen B. Identification of novel microRNA regulatory pathways associated with heterogeneous prostate cancer. BMC Syst Biol. 2013;7(Suppl 3):S6.

14. Lin Y, Chen J, Shen B. Interactions between genetics, lifestyle, and environmental factors for healthcare. Adv Exp Med Biol. 2017;1005:167-91.

15. Yan W, Wang S, Sun Z, Lin Y, Sun S, Chen J, Chen W. Identification of microRNAs as potential biomarker for gastric cancer by system biological analysis. Biomed Res Int. 2014;2014:901428.

16. Zhao XM, Liu KQ, Zhu G, He F, Duval B, Richer JM, Huang DS, Jiang CJ, Hao JK, Chen L. Identifying cancer-related microRNAs based on gene expression data. Bioinformatics. 2015;31:1226-34

17. Sun SY, Liu ZP, Zeng T, Wang Y, Chen L. Spatio-temporal analysis of type 2 diabetes mellitus based on differential expression networks. Sci Rep. 2013;3:2268.

18. Cogill S, Wang L. Support vector machine model of developmental brain gene expression data for prioritization of Autism risk gene candidates. Bioinformatics. 2016;32:3611-8.

19. Zhang W, Edwards A, Fan W, Flemington EK, Zhang K. miRNA-mRNA correlation-network modules in human prostate cancer and the differences between primary and metastatic tumor subtypes. PLOS ONE. 2012;7:e40130.

20. Zhang W, Zang J, Jing X, Sun Z, Yan W, Yang D, Shen B, Guo F. Identification of candidate miRNA biomarkers from miRNA regulatory network with application to prostate cancer. J Transl Med. 2014;12:66.

21. Yan W, Xu L, Sun Z, Lin Y, Zhang W, Chen J, Hu S, Shen B. MicroRNA biomarker identification for pediatric acute myeloid leukemia based on a novel bioinformatics model. Oncotarget. 2015;6:26424-36.

22. Zhu J, Wang S, Zhang W, Qiu J, Shan Y, Yang D, Shen B. Screening key microRNAs for castration-resistant prostate cancer based on miRNA/ mRNA functional synergistic network. Oncotarget. 2015;6:43819-30.

23. Taylor BS, Schultz N, Hieronymus H, Gopalan A, Xiao Y, Carver BS, Arora VK, Kaushik P, Cerami E, Reva B, et al. Integrative genomic profiling of human prostate cancer. Cancer Cell. 2010;18:11-22.

24. Varambally S, Yu J, Laxman B, Rhodes DR, Mehra R, Tomlins SA, Shah RB, Chandran U, Monzon FA, Becich MJ, et al. Integrative genomic and proteomic analysis of prostate cancer reveals signatures of metastatic progression. Cancer Cell. 2005;8:393-406.

25. Edgar R, Domrachev M, Lash AE. Gene expression omnibus: NCBI gene expression and hybridization array data repository. Nucleic Acids Res. 2002;30:207-10.

26. Peng X, Guo W. Altered expression of microRNAs in bone metastasis compared with human prostate cancer. 2012. https://www.ncbi.nlm.nih. gov/geo/query/acc.cgi?acc=GSE26964. Accessed 10 Apr 2018.

27. Vanacore D, Boccellino M, Rossetti S, Cavaliere C, D'Aniello C, Di Franco R, Romano FJ, Montanari M, La Mantia E, Piscitelli R, et al. Micrornas in prostate cancer: an overview. Oncotarget. 2017;8:50240-51.

28. Ritchie ME, Phipson B, Wu D, Hu Y, Law CW, Shi W, Smyth GK. limma powers differential expression analyses for RNA-sequencing and microarray studies. Nucleic Acids Res. 2015:43:e47.

29. Smyth GK. Linear models and empirical bayes methods for assessing differential expression in microarray experiments. Stat Appl Genet Mol Biol. 2004:3:1-25.

30. Benjamini Y, Hochberg Y. Controlling the false discovery rate-a practical and powerful approach to multiple testing. J R Stat Soc Ser B Methodol. 1995;57:289-300

31. Kozomara A, Griffiths-Jones S. miRBase: annotating high confidence microRNAs using deep sequencing data. Nucleic Acids Res. 2014:42:D68-73.

32. Hsu SD, Tseng YT, Shrestha S, Lin YL, Khaleel A, Chou CH, Chu CF, Huang HY, Lin CM, Ho SY, et al. miRTarBase update 2014: an information resource for experimentally validated miRNA-target interactions. Nucleic Acids Res. 2014:42:D78-85.

33. Vergoulis T, Vlachos IS, Alexiou P, Georgakilas G, Maragkakis M, Reczko M, Gerangelos S, Koziris N, Dalamagas T, Hatzigeorgiou AG. TarBase 6.0: 
capturing the exponential growth of miRNA targets with experimental support. Nucleic Acids Res. 2012;40:D222-9.

34. Xiao F, Zuo Z, Cai G, Kang S, Gao X, Li T. miRecords: an integrated resource for microRNA-target interactions. Nucleic Acids Res. 2009;37:D105-10.

35. Jiang $Q$, Wang Y, Hao Y, Juan L, Teng M, Zhang X, Li M, Wang G, Liu Y. miR2Disease: a manually curated database for microRNA deregulation in human disease. Nucleic Acids Res. 2009;37:D98-104.

36. Gennarino VA, Sardiello M, Mutarelli M, Dharmalingam G, Maselli V, Lago $\mathrm{G}$, Banfi S. HOCTAR database: a unique resource for microRNA target prediction. Gene. 2011;480:51-8.

37. Gamazon ER, Im HK, Duan S, Lussier YA, Cox NJ, Dolan ME, Zhang W. Exprtarget: an integrative approach to predicting human microRNA targets. PLoS ONE. 2010;5:e13534.

38. Li JH, Liu S, Zhou H, Qu LH, Yang JH. starBase v2.0: decoding miRNAceRNA, miRNA-ncRNA and protein-RNA interaction networks from largescale CLIP-Seq data. Nucleic Acids Res. 2014;42:D92-7.

39. Chongsuvivatwong V. Epicalc: epidemiological calculator, R package version 2.15.1.0. 2012. https://cran.r-project.org/src/contrib/Archive/epicalc/. Accessed 18 Apr 2018

40. Kanehisa M, Goto S. KEGG: kyoto encyclopedia of genes and genomes. Nucleic Acids Res. 2000;28:27-30.

41. Kramer A, Green J, Pollard J Jr, Tugendreich S. Causal analysis approaches in ingenuity pathway analysis. Bioinformatics. 2014;30:523-30.

42. da Huang W, Sherman BT, Lempicki RA. Systematic and integrative analysis of large gene lists using DAVID bioinformatics resources. Nat Protoc. 2009;4:44-57.

43. Watahiki A, Wang Y, Morris J, Dennis K, O'Dwyer HM, Gleave M, Gout PW, Wang Y. MicroRNAs associated with metastatic prostate cancer. PLoS ONE. 2011;6:e24950.

44. Pang Y, Young CY, Yuan H. MicroRNAs and prostate cancer. Acta Biochim Biophys Sin. 2010:42:363-9.

45. Huang S, Guo W, Tang Y, Ren D, Zou X, Peng X. miR-143 and miR-145 inhibit stem cell characteristics of PC-3 prostate cancer cells. Oncol Rep. 2012;28:1831-7.

46. Ren D, Wang M, Guo W, Zhao X, Tu X, Huang S, Zou X, Peng X. Wild-type p53 suppresses the epithelial-mesenchymal transition and stemness in PC-3 prostate cancer cells by modulating miR145. Int J Oncol. 2013:42:1473-81.

47. Chakravarthi BV, Goswami MT, Pathi SS, Robinson AD, Cieslik M, Chandrashekar DS, Agarwal S, Siddiqui J, Daignault S, Carskadon SL, et al. MicroRNA-101 regulated transcriptional modulator SUB1 plays a role in prostate cancer. Oncogene. 2016:35:6330-40.

48. Lin YC, Lin JF, Tsai TF, Chou KY, Chen HE, Hwang TI. Tumor suppressor miRNA-204-5p promotes apoptosis by targeting BCL2 in prostate cancer cells. Asian J Surg. 2017:40:396-406.

49. Todorova K, Metodiev MV, Metodieva G, Zasheva D, Mincheff M, Hayrabedyan S. miR-204 is dysregulated in metastatic prostate cancer in vitro. Mol Carcinog. 2016;55:131-47.

50. Theodore SC, Davis M, Zhao F, Wang H, Chen D, Rhim J, Dean-Colomb W, Turner T, Ji W, Zeng G, et al. MicroRNA profiling of novel African American and Caucasian Prostate Cancer cell lines reveals a reciprocal regulatory relationship of miR-152 and DNA methyltranferase 1. Oncotarget. 2014:5:3512-25.

51. Walter BA, Valera VA, Pinto PA, Merino MJ. Comprehensive microRNA profiling of prostate cancer. J Cancer. 2013:4:350-7.

52. Lakshman M, Huang X, Ananthanarayanan V, Jovanovic B, Liu Y, Craft CS, Romero D, Vary CP, Bergan RC. Endoglin suppresses human prostate cancer metastasis. Clin Exp Metastasis. 2011;28:39-53.

53. Yan Z, Jin $S$, Wei Z, Huilian H, Zhanhai Y, Yue T, Juan L, Jing L, Libo Y, Xu L. Discoidin domain receptor 2 facilitates prostate cancer bone metastasis via regulating parathyroid hormone-related protein. Biochim Biophys Acta. 2014;1842:1350-63.

54. Caley MP, King H, Shah N, Wang K, Rodriguez-Teja M, Gronau JH, Waxman J, Sturge J. Tumor-associated Endo180 requires stromal-derived LOX to promote metastatic prostate cancer cell migration on human ECM surfaces. Clin Exp Metastasis. 2016:33:151-65.

55. Sun GG, Wang YD, Cui DW, Cheng YJ, Hu WN. EMP1 regulates caspase-9 and VEGFC expression and suppresses prostate cancer cell proliferation and invasion. Tumour Biol. 2014;35:3455-62.
56. Noh BJ, Sung JY, Kim YW, Chang SG, Park YK. Prognostic value of ERG, PTEN, CRISP3 and SPINK1 in predicting biochemical recurrence in prostate cancer. Oncol Lett. 2016;11:3621-30.

57. Nowak DG, Cho H, Herzka T, Watrud K, DeMarco DV, Wang VM, Senturk S, Fellmann C, Ding D, Beinortas T, et al. MYC drives Pten/Trp53-deficient proliferation and metastasis due to IL6 secretion and AKT suppression via PHLPP2. Cancer Discov. 2015;5:636-51.

58. Xu B, Tao T, Wang Y, Fang F, Huang Y, Chen S, Zhu W, Chen M. hsa-miR135a-1 inhibits prostate cancer cell growth and migration by targeting EGFR. Tumour Biol. 2016;37:14141-51.

59. Pan XW, Gan SS, Ye JQ, Fan YH, Hong U, Chu CM, Gao Y, Li L, Liu X, Chen L, et al. SMC1A promotes growth and migration of prostate cancer in vitro and in vivo. Int J Oncol. 2016;49:1963-72.

60. Zhang SR, Yang JK, Xie JK, Zhao LC. Long noncoding RNA HOTTIP contributes to the progression of prostate cancer by regulating HOXA13. Cell Mol Biol. 2016;62:84-8.

61. Wang X, Wen J, Li R, Qiu G, Zhou L, Wen X. Gene expression profiling analysis of castration-resistant prostate cancer. Med Sci Monit. 2015;21:205-12.

62. Destouches D, Sader M, Terry S, Marchand C, Maille P, Soyeux P, Carpentier G, Semprez F, Ceraline J, Allory Y, et al. Implication of NPM1 phosphorylation and preclinical evaluation of the nucleoprotein antagonist N6L in prostate cancer. Oncotarget. 2016;7:69397.

63. Grubb RL, Deng J, Pinto PA, Mohler JL, Chinnaiyan A, Rubin M, Linehan WM, Liotta LA, Petricoin EF, Wulfkuhle JD. Pathway biomarker profiling of localized and metastatic human prostate cancer reveal metastatic and prognostic signatures. J Proteome Res. 2009:8:3044-54.

64. Xiao GQ, Unger P, Yang Q, Kinoshita Y, Singh K, McMahon L, Nastiuk K, Sha K, Krolewski J, Burstein D. Loss of PLZF expression in prostate cancer by immunohistochemistry correlates with tumor aggressiveness and metastasis. PLOS ONE. 2015:10:e0121318.

65. Ding Y, He D, Florentin D, Frolov A, Hilsenbeck S, Ittmann M, Kadmon D, Miles B, Rowley D, Ayala G. Semaphorin 4F as a critical regulator of neuroepithelial interactions and a biomarker of aggressive prostate cancer. Clin Cancer Res. 2013:19:6101-11.

66. McNair C, Urbanucci A, Comstock CE, Augello MA, Goodwin JF, Launchbury R, Zhao SG, Schiewer MJ, Ertel A, Karnes J, et al. Cell cycle-coupled expansion of AR activity promotes cancer progression. Oncogene. 2016;36:1655-68.

67. Zhu W, Xue Y, Liang C, Zhang R, Zhang Z, Li H, Su D, Liang X, Zhang Y, Huang Q, et al. S100A16 promotes cell proliferation and metastasis via AKT and ERK cell signaling pathways in human prostate cancer. Tumour Biol. 2016;37:12241-50.

68. Lim W, Park S, Bazer FW, Song G. Naringenin-induced apoptotic cell death in prostate cancer cells is mediated via the PI3K/AKT and MAPK signaling pathways. J Cell Biochem. 2016;118:1118-31.

69. Browne AJ, Gobel A, Thiele S, Hofbauer LC, Rauner M, Rachner TD. p38 MAPK regulates the Wnt inhibitor Dickkopf-1 in osteotropic prostate cancer cells. Cell Death Dis. 2016;7:e2119.

70. Wang J, Kobayashi T, Floc'h N, Kinkade CW, Aytes A, Dankort D, Lefebvre C, Mitrofanova A, Cardiff RD, McMahon M, et al. B-Raf activation cooperates with PTEN loss to drive c-Myc expression in advanced prostate cancer. Cancer Res. 2012;72:4765-76.

71. Chen H, Zhou L, Wu X, Li R, Wen J, Sha J, Wen X. The PI3K/AKT pathway in the pathogenesis of prostate cancer. Front Biosci. 2016;21:1084-91.

72. Kassi E, Moutsatsou P. Glucocorticoid receptor signaling and prostate cancer. Cancer Lett. 2011;302:1-10.

73. Massague J, Gomis RR. The logic of TGFbeta signaling. FEBS Lett. 2006;580:2811-20.

74. Li S, Lv Q, Sun H, Xue Y, Wang P, Liu L, Li Z, Li Z, Tian X, Liu YH. Expression of TRAP1 predicts poor survival of malignant glioma patients. J Mol Neurosci. 2015;55:62-8.

75. Bartsch DK, Fendrich V, Slater EP, Sina-Frey M, Rieder H, GreenhalfW, Chaloupka B, Hahn SA, Neoptolemos JP, Kress R. RNASEL germline variants are associated with pancreatic cancer. Int J Cancer. 2005;117:718-22.

76. Gupta GP, Massague J. Cancer metastasis: building a framework. Cell. 2006;127:679-95.

77. Lin Y, Qian F, Shen L, Chen F, Chen J, Shen B. Computer-aided biomarker discovery for precision medicine: data resources, models and applications. Brief Bioinform. 2017. https://doi.org/10.1093/bib/bbx158. 
78. Heger Z, Merlos Rodrigo MA, Michalek P, Polanska H, Masarik M, Vit V, Plevova M, Pacik D, Eckschlager T, Stiborova M, Adam V. Sarcosine up-regulates expression of genes involved in cell cycle progression of metastatic models of prostate cancer. PLoS ONE. 2016;11:e0165830

79. Erdmann K, Kaulke K, Rieger C, Salomo K, Wirth MP, Fuessel S. MiR$26 a$ and miR-138 block the G1/S transition by targeting the cell cycle regulating network in prostate cancer cells. J Cancer Res Clin Oncol. 2016;142:2249-61.

80. Fu W, Madan E, Yee M, Zhang H. Progress of molecular targeted therapies for prostate cancers. Biochim Biophys Acta. 2012;1825:140-52.

Ready to submit your research? Choose BMC and benefit from:

- fast, convenient online submission

- thorough peer review by experienced researchers in your field

- rapid publication on acceptance

- support for research data, including large and complex data types

- gold Open Access which fosters wider collaboration and increased citations

- maximum visibility for your research: over 100M website views per year

At BMC, research is always in progress.

Learn more biomedcentral.com/submissions 